\title{
Mouse Genetics 2016: meeting report
}

\author{
Jordana C. Bloom ${ }^{1} \cdot$ Tina N. Tran ${ }^{1} \cdot$ Robert J. Yamulla ${ }^{1}$
}

Received: 17 September 2016 / Accepted: 10 February 2017 / Published online: 22 February 2017

(C) Springer Science+Business Media New York 2017

\section{Introduction}

This year's Mouse Genetics Meeting was a combination of the 30th International Mammalian Genome Conference (IMGC) and the Mouse Molecular Genetics (MMG) Meeting. The meeting was held from July 13-17, 2016 in Orlando, Florida as part of The Allied Genetics Conference (TAGC) where in addition to the Mouse community, geneticists from the C. elegans, ciliate, Drosophila, yeast, zebrafish, and population, evolutionary and quantitative genetics communities were present. TAGC celebrated the 100th anniversary of the research journal, Genetics, which has been publishing landmark studies in model organism genetics since its inception. The joint conference between the mouse communities and the other model organism communities present at the conference allowed for collaboration and discourse among researchers who typically attend separate meetings. Cross-community dialogue was fostered by the TAGC app that allowed attendees to design their own schedules based on research themes.

This report focuses on the Mouse Genetics meeting, which continued where the successful IMGC in Yokohama, Japan left off (Gonzales et al. 2016). The meeting spanned ten sessions with a diverse array of speakers, including a symposium to highlight trainees and their research. Sessions included talks on new resources available to the mouse genetics community, in addition to ongoing research in many areas, including but not limited to comparative

All authors contributed equally to the work.

\section{Jordana C. Bloom}

1 Department of Molecular Biology and Genetics, Cornell University, Ithaca, NY 14850, USA genomics, evolution, development, epigenetics, and human disease models. The research presented from each of the sessions will be highlighted below. Mouse Genetics 2016 was organized by Teresa Gunn and Monica Justice, with assistance from David Beier, Martin Hrabé de Angelis, Yumiko Saga, Philippe Soriano, and François Spitz, as well as invaluable help from Darla Miller. The meeting program and abstracts are available online at http://www.genetics2016.org/presenters/abstracts.

\section{Trainee Symposium}

IMGC has traditionally focused on trainee education, and at this year's meeting, there were 16 students and post-doctoral associates selected to present their projects during the Mouse Trainee Symposium. Of the 16 presentations, five received the Lorraine Flaherty Trainee Speaking Award: Meng Zhang, Jacob Moskowitz, Nithya Kartha, Anthony Doran, and Elijah Edmondson. The breadth of topics covered by trainees extended from neurogenesis to deep space radiation exposure, highlighting the increasingly diverse interests and innovative projects emerging in the field of mouse genetics.

Meng Zhang (Yale University) reported on her progress in characterizing the role of PUF family proteins in neurogenesis. Although the PUF proteins are known to act in germline stem cell maintenance in Drosophila and $C$. elegans and in spermatogenesis in mice, their role in neurogenesis remains undefined. Her investigation currently focuses on Pum 1 and Pum2, where mutant models suggest that the genes play a role in regulating neurogenesis through a RNA-regulating mechanism.

Jacob Moskowitz (University of Missouri) and Nithya Kartha (Cornell University) focused on cancer biology 
with talks relevant to colon and breast cancer, respectively. Moskowitz looks at irregularities in colon cancer incidence using $A p c^{M i n}$ mice from different colonies and asks whether incidence is modulated by environmental effects. His results suggest that cancer incidence is largely influenced by gut microbiota composition, which could explain years of inconsistencies among colon cancer mouse studies. Kartha's talk centered around characterization of Aridla, a member of the SWI/SNF chromatin remodeling complex, as a suppressor of spontaneous mammary tumors in mice. Kartha provided evidence on Aridla functioning as a tumor suppressor, showing that reintroduction of its expression in tumorigenic lines impairs tumor formation in the mammary fat pad. Her future work will focus on CRISPR-activation of silenced Aridla alleles, if successful, could be a novel approach in treating breast cancer.

Genome-wide approaches were utilized by Anthony Doran (Wellcome Trust Sanger Institute) and Elijah Edmondson (Colorado State University) in their projects. Doran applies deep genome sequencing to define the genetic source of variation among 13 inbred mouse strains, identifying 27 million SNPs and 4.36 million insertion-deletion events. Of the variants, 2.79 million SNPs and 0.56 million insertion-deletions were novel. Lastly, Edmondson is studying HZE-ion radiation, which is commonly found outside of the Earth's atmosphere. NASA's current risk model uses gamma radiation as a surrogate, and Edmondson asks whether the two forms of radiation-induced DNA damage are comparable. The experimental design includes 615 outbred mice per group, where groups were exposed to gamma irradiation, 0.4 Gy HZE-ion radiation, or left unirradiated. In a massive undertaking, the mice were housed throughout their lifespans and monitored for disease development, and were ultimately sequenced for associated variants and damage-susceptible loci. Fortunately, NASA's current risk model was validated, as Edmondson found significant overlap between the consequences of HZEion- and gamma irradiation-induced damage.

In addition to the Mouse Trainee Symposium, three poster sessions showcased 112 posters by trainees, allowing for the mouse genetics community to share work with all attendees at the conference. The recipients of awards for outstanding oral and poster presentations are listed in Table 1.

\section{International resources and technological innovations}

Reports on technological innovation and research resources have been a central aspect of previous IMGC meetings and this year was no exception. A broad range of topics were covered, with an overarching theme of database construction and refinement, embryonic phenotyping, and CRISPR/Cas9 genome editing.

Martin Hrabé de Angelis (Institute of Experimental Genetics-Germany) began the International Resources session by describing challenges associated with mammalian genome annotation and mutant phenotyping. Despite these difficulties, Hrabé de Angelis reported near completion of Phase I genome annotation by the International Mouse Phenotyping Consortium (IMPC). Nearly 5000 mutant mouse lines were characterized through a standardized phenotyping pipeline. Upon completion of Phase I, the IMPC will begin work on analyzing the remaining protein-coding genes for Phase II. Presentations by Stephen Murray (The Jackson Laboratory) and Jacqui White (The Francis Crick Institute) complemented the IMPC report by detailing their work on characterizing embryonic lethal phenotypes in mice. Of the near 5000 genes reported by IMPC, 400 demonstrate embryonic lethality. Techniques such as high-resolution episcopic microscopy on tissue were used to characterize each specimen, resulting in analysis of over 300 embryos and production of three million images. 3D imaging technologies were discussed further in the Technological Innovations session by Mary Dickinson (Baylor College of Medicine), along with their applicability to the IMPC project and Knockout Mouse Project 2 (KOMP2). Current embryonic data can be accessed for free on DMDD.org. uk and mouse lines can be acquired through the European Mutant Mouse Archive (EMMA).

GENCODE, an encyclopedia of human gene structures and variants, has also benefited from recent technological breakthroughs. Mark Thomas (Wellcome Trust Sanger Institute) described their combinatorial use of RNA-seq, CAGE, Poly-A seq, phyloCSF, and mass spectrometry to study transcribed regions of the genome. Importantly, these methods refined the former list of mouse coding genes. His findings decreased the total number of coding genes and added previously unidentified pseudogenes and long noncoding RNA transcripts.

CRISPR/Cas9 genome editing was a prominent feature of the Technological Innovation session as well, where current advancements and novel applications of the technology were described by Prem Premsrirut (Mirimus, Inc), Channabasavaiah Gurumurthy (University of Nebraska Medical Center), and Thom Saunders (University of Michigan). Premsrirut outlined a novel method for using CRISPR/ Cas 9 and RNAi as an affordable means for assessing potential drug targets. In this system, disease-sensitized alleles are created via CRISPR/Cas9 gene editing, while in parallel, Tet-inducible RNAi is used to determine whether knockdown of other genes can rescue or mitigate CRISPRinduced disease phenotypes. Gurumurthy and Saunders focused on current CRISPR methodologies and their effect on genome editing success rates. Specifically, Gurumurthy 


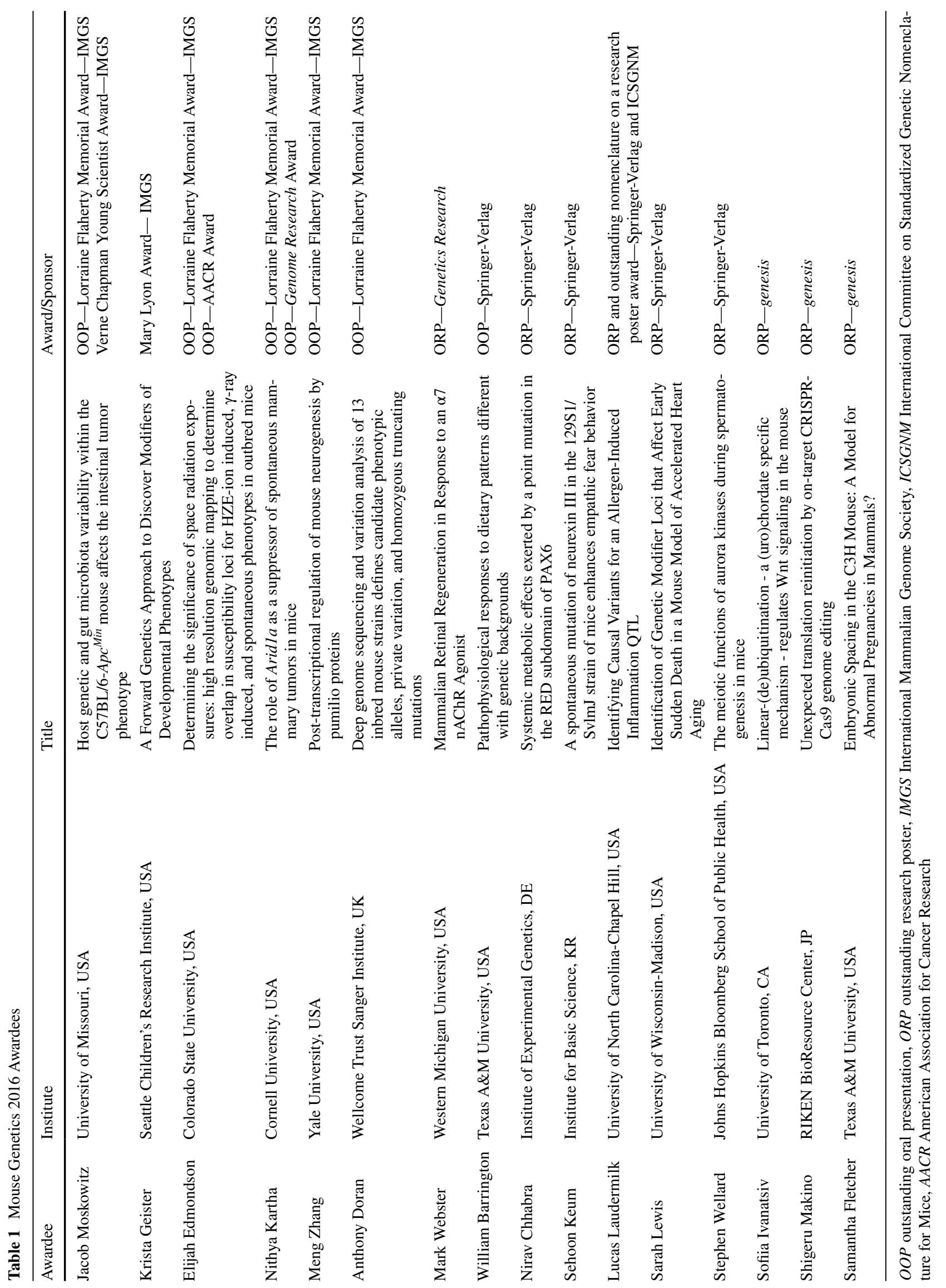


argued that providing a single-stranded DNA template may address the "CRISPR imbalance," or the tendency for editing to result in error-prone repair rather than homology-directed repair. Gurumurthy reports that homologydirected repair and preferred allele integration can increase up to $25 \%$ when single-stranded DNA repair templates are used instead of double-stranded templates, regardless of sequence length.

While nearly all presenters discussed mouse models, Elizabeth Bryda's talk emphasized the benefit of rat models in complementing mouse studies. Bryda described a novel cell ablation system where target cells express CD59 membrane receptor that can be selectively bound by intermedilysin (ILY), resulting in cell ablation. CD59 expression was demonstrated successfully in various tissues, allowing for user-defined ablation of a tissue or cell type. Her talk encourages the use of this technology in studying anemia, liver damage, and neuronal damage.

\section{Comparative genomics, computational methods, and evolution}

The ability to collect and analyze increasingly large datasets has allowed for the rapid advancement and growth of the comparative genomics and population genetics fields. Hopi Hoekstra (Howard Hughes Medical Institute (HHMI)/ Harvard University) began this session by discussing a genetic screen comparing monogamous and promiscuous deer mice to identify loci associated with differences in the species' prenatal care. She identified vasopressin as a locus of particular interest due to high expression in promiscuous species. This discovery may explain the tendency for promiscuous species to build less sophisticated nests for their young. Yoichi Gondo (Riken BioResource Center) reported on the results of a breeding scheme for generating germline mutations. Using whole genome sequencing, he identified single-nucleotide variants that arise de novo between G1 gametogenesis and G5 fertilization in mice.

Complementary talks from Thomas Keane and Jingtao Li (Keane group, Wellcome Trust Sanger Institute) described the use of genome-wide technologies for sequencing 16 mouse strains, including laboratory and wild-derived strains. Currently, they have discovered over 100 subspecies-specific haplotypes. Their data suggest that highly polymorphic loci of wild-derived strains are enriched for immunity, programmed cell death, and sensory functions, possibly explaining why phenotypic responses vary among mice. Gary Churchill (The Jackson Laboratory) ended the session with work combining RNAseq and shotgun proteomics to address why transcript- and protein-level variations occur at different loci. Using Diversity Outbred (DO) mice, their study found two classes of gene regulation. A majority of protein abundance QTLs (pQTLs) map locally to a gene and act in cis to influence message and protein levels. In contrast, distant pQTLs have low correlation with expression due to a trans-acting, posttranslational mechanism that involves stoichiometric buffering of protein complexes and metabolic pathways.

\section{Development and morphogenesis}

Both development and morphogenesis are highly dynamic; thus, studying their progression can be challenging. In the first talk, Takashi Hiiragi (EMBL-Germany) discussed a novel imaging approach to capture stages of embryogenesis with spatio-temporal resolution. With it, his group studies how polarity is established in mammalian eggs. For example, cells that receive higher cortical tension are more likely to adopt an inner cell mass versus trophectoderm cell fate. Nicolas Plachta (EMBL-Australia and the Agency for Science Technology and Research) continued the discussion with his approach to live-cell imaging, where he labels transcription factors with photoactivatable GFP in order to measure transcription factor-DNA binding events in developing mouse embryos. He found that in the case of factors POU5F1 (OCT4) and SOX2, their binding specificities to certain DNA sites significantly change as cells adopt their cell fates.

Neil Hunter (HHMI/University of California-Davis) described how SUMO, ubiquitins, and proteasomes mediate the events of meiotic prophase, such as synapsis and DNA recombination. His work revealed that proper crossing-over depends on regulated protein degradation at chromosome axes, where RNF212-dependent SUMO can stall recombination by mediating degradation of recombination factors through ubiquitylation. Grace Hwang (Johns Hopkins Bloomberg School of Public Health) also discussed regulation of meiosis with her work examining the SMC5/6 complex in oocytes. She found that $\mathrm{Smc5}$ conditional knockout oocytes are incapable of homologous chromosome segregation during meiosis I, leading to aneuploidy in meiosis II. Following conditional deletion, she observed that depletion of the complex diminishes with age, leading to a model connecting SMC5/6 complex levels with ageassociated meiotic abnormalities.

Jadiel Wasson (Emory University School of Medicine) found that loss of maternal KDM1A leads to embryonic arrest at the 1- to 2-cell stage, due to an inability to undergo the maternal-to-zygotic transition. Additionally, she showed that partial loss of maternal KDM1A leads to abnormal behavior in adult progeny, stemming from alterations in DNA methylation and expression of imprinted genes. Krista Geister (Seattle Children's Research Institute) described a novel method for mapping causal ENU-induced 
mutations that does not require strain outcrossing. Using this method, she conducted a screen to look for modifiers of developmental phenotypes among inbred mouse strains. To conclude the session, Clement Chow (University of Utah) discussed the effects of endoplasmic reticulum (ER) stress on placental mRNA and small RNA levels. Using RNAseq, he found that the placenta has a different response to ER stress between E10.5 and E14.5. Future work in his lab will continue to explore these variations.

\section{Rosa Beddington Lecture}

This year's meeting once again honored the life and work of Rosa Beddington through the Rosa Beddington Lecture. Beddington was well known for her substantial contributions to the field of developmental genetics through her study of axial patterning, germ layer specification, and gastrulation. This year's lecture was given by Anna-Katerina Hadjantonakis (Memorial Sloan Kettering Cancer Center). Hadjantonakis began by noting the past twenty years of advancements, such as the work of many geneticists such as Rosa Beddington to identify genes that regulate major developmental processes in mice. Despite incredible progress, she believes that there is still more to be done, especially toward understanding the interplay between genes and the development of tissues and organs in mammals. Her lab is working to address this knowledge gap using high-resolution quantitative microscopy and gene expression profiling to study cell lineage specification and tissue patterning during blastocyst development and gastrulation.

\section{Stem cells}

Following the Rosa Beddington Lecture, Helen Abud (Monash Biomedicine Discovery Institute) continued the session by presenting her work on intestinal epithelium crypt base columnar (CBC) stem cells. Her studies show that loss of Snail results in decrease CBC stem cell proliferation and apoptosis, while ectopic expression leads to an increase in proliferation. Most strikingly, cells with Snail depleted do not proliferate in response to radiation-induced damage, highlighting an integral role of Snail in regeneration of the intestinal epithelium in response to damage. Christoph Lepper (Carnegie Institution for Science) described his work examining muscle fiber regeneration using the TEAD1 transgenic mouse model. After inducing muscle injury using $\mathrm{BaCl}_{2}$ derived from snake venom, he showed that muscle stem cells are responsible for increased muscle regeneration in the transgenic model.
Joshua Brickman (University of Copenhagen) concluded the Stem Cells session by discussing how embryonic stem cells (ESCs) are able to maintain their developmental plasticity even though they have undergone a certain level of patterning and/or differentiation. His research revealed two new mechanisms allowing for the maintenance of plasticity in ESCs. The first mechanism involves the polycomb repressor complex 2 (PRC2), which allows transcriptional stimulation and patterning without lineage commitment. The second mechanism depends on the ability of RNA polymerase II to reversibly disengage from promoters in response to signaling cues. Both mechanisms allow ESCs to retain their ability to self-renew while remaining primed for differentiation.

\section{Epigenetics}

A popular topic at this year's Epigenetics session was application of current genome- and transcriptome-wide technologies to study basic biology and disease. Jeannie Lee (Massachusetts General Hospital) opened with epigenetic regulation at the RNA-chromatin interface. Lee added to the known roles of long non-coding RNAs (lncRNAs) by demonstrating their involvement in protein recruitment, $\mathrm{X}$-chromosome counting, allele choice, and X-inactivation. Her presentation provided a foundation for Stefan Pinter's (University of Connecticut Health Center) discussion on monoallelic gene expression. Pinter found that about $20 \%$ of the mouse transcriptome exhibits monoallelic expression and that this effect exhibits tissue-specific variation. Furthermore, Pinter observed that genotype could significantly influence the penetrance of parental alleles. Gregory Carter (The Jackson Laboratory) also studies the effect of genetic variation of regulatory regions. His research combined RNA-seq, bisulfite DNA-seq, and ChIP-seq to characterize H3K4me1, H3Lys4me3, and H3K27ac epigenetic markers. Interestingly, Carter found mouse strain-specific differences in both gene expression and epigenetic markers and hypothesizes that such differences between individuals may confer divergent responses to drug therapy.

Nora Engel (Temple University) and Christopher Baker (The Jackson Laboratory) presented work on sex-specific differences in mouse ESCs and cis- versus trans-control of the epigenetic landscape, respectively. Engel hypothesized that discrepancies in male and female mortality rates cannot be due to hormones alone. Using RNA-seq on XX an XY embryonic ESCs, she found that 400 coding genes and 300 non-coding RNAs varied between the sexes. These findings will serve as the basis for future work on understanding disease origin in both genders. Baker presented his work that investigates genomic levels of $\mathrm{H} 3 \mathrm{~K} 4 \mathrm{me} 3$, an epigenetic marker associated with promoters, enhancers, 
and recombination hotspots. Comparing C57BL6/J and DBA2/J mouse spermatocytes, he found that H3K4me3 levels varied significantly between the two strains. Furthermore, F1 hybrids showed evidence of trans-control at multiple sites, where phenotype did not match the genotype of the parent.

The session also featured David Katz (Emory University), who investigates the consequence of amyloid- $\beta$ and pTau aggregation in relation to Alzheimer's Disease. Amyloid- $\beta$ and pTau aggregation results in KDM1 A/LSD1 histone demethylase mislocalization, which may interfere with its normal epigenomic regulatory roles. Indeed, loss of Kdmla resulted in Alzheimer's-like symptoms in mice, suggesting that KDM1A activity is important in Alzheimer's prevention. Finally, Weipeng Mu (University of North Carolina at Chapel Hill) reported that isoforms of polycomb-repressive complex 2 subunit EZHZ2 have distinctly different functions in non-dividing cells, such as spermatocytes. Such differences would alter epigenetic regulation of stem cell pluripotency and somatic differentiation.

\section{Translational and System Genetics}

The Translational and System Genetics session began with Monica Justice (Hospital for Sick Children), who is working on a genetic suppressor screen to identify mutations associated with Rett Syndrome. Her current findings reveal that mutations in cholesterol synthesis and lipid homeostasis pathways are promising targets for drug therapy. Also studying Rett Syndrome, Lucy Williams (University of North Carolina) is combining genomic, proteomic, and epigenetic methods to study how transcript expression and protein levels are maintained in the brain. Using a variety of diverse mouse strains, she found that genetic background is a major factor behind why severity of neuronal diseases differs from patient to patient.

Steven Munger (The Jackson Laboratory) investigates why transcript and protein levels correlate in most tissues yet found to be inconsistent in some other cases. Using RNA-seq and shotgun proteomics, he described two classes of gene regulation to explain these phenomena: (1) generally, a quantitative trait locus (QTL) maps locally to the gene region and acts in cis to affect both transcription and protein response, while (2) a distal QTL is trans-acting and is uncoupled from both responses. Lastly, Jean Winter (National Human Genome Research Institute) spoke on the importance of improving current methods for diagnosis and treatment of disease, especially in the case of prostate cancer. Prostate cancer is commonly misdiagnosed and overtreated; therefore, research on loci associated with increased susceptibility for disease initiation and development are paramount. Her current work includes characterization of two novel prostate cancer susceptibility genes: Cenpu and Rwdd4.

\section{Human disease models}

This year's conference held two sessions featuring the use of mice for human disease modeling. Mouse neurological studies were highlighted in the first session, as Freda Miller (The Hospital for Sick Children) discussed an alternative use for the diabetic drug, metformin, to promote proliferation and differentiation of neural precursor cells. Her results suggest that metformin has the potential to treat patients with previous brain lesions. Continuing along the neuroscience theme, Michelle Southard-Smith (Vanderbilt University Medical School) genetically engineered a mouse carrying an inducible Sox10 dominant-negative allele that allows her group to study phenotypes prevalent among patients with a range of neurocristopathies. Additionally, Deborah Cabin (McLaughlin Research Institute) presented work on characterizing the protein alpha-synuclein, which appears to be toxic at high levels and may contribute to the onset of Parkinson's disease.

The session continued with mouse models for studying blood disease and malaria. Danitza Nebor (The Jackson Laboratory) performed RNA-seq to identify genes associated with the transcription factor Klfl, which has been previously linked to lifelong anemia in heterozygous mice. Gaetan Burgio (Australian National University) performed an ENU mutagenesis screen to find protective mutations from malaria infection. Ultimately, mutations for host-resistance could serve as potential targets for drug development. Later presentations switched the session's focus toward molecular interactions, where Suzanne Hartford (National Cancer Institute) showed that the interaction between BRCA2 and its localizing protein, PALB2, is essential for maintaining genome integrity. Finally, William Barrington (North Carolina State University) presented work on how the effects of diet vary between mouse strains. By comparing the Western diet to traditional Japanese or ketogenic diets, he showed that different strains had distinct physiological responses, where variations included changes in adiposity, glucose tolerance, and metabolic rate. His results argue against promoting a universal diet model for optimal health and suggest that the "ideal" diet varies between individuals.

Laura Reinholdt (The Jackson Laboratory) began the second session by describing a large-scale study to identify naturally occurring disease mutations in multiple mouse strains using whole genome sequencing. Currently, this work has discovered over 90 new disease-causing alleles, where $10 \%$ of mutations occur in novel genes. Tania Sorg 
(PHENOMIN-Institut Clinique de la Souris) is characterizing a mouse carrying a G12S mutation in the Hras gene. These mice display phenotypes seen in patients with Costello Syndrome, such as reduced motor activity and muscle strength, and can serve as a proxy for future therapy development. David Beier (Seattle Children's Research Institute) continued the talks with his study on brain lamination defects, where his current work focuses on reelin expression and its role in ataxia development. Finally, Miriam Meisler (University of Michigan) shared work on Scn8a, a gene encoding a sodium channel involved in generating action potentials in neurons. Her group has recently discovered a mutation in $S c n 8 a$ that may be linked to epileptic encephalopathy.

Diseases in bone are also areas of active research. Aris Economides (Regeneron Pharmaceuticals) is studying fibrodysplasia ossificans progressiva (FOP), a debilitating disease that causes immobility in patients. FOP is associated with mutations in Acvrl and current work is being done to inhibit and even reverse ossification by inhibiting activin A. Chris Lelliot (Wellcome Trust Sanger Institute) is working on high-throughput phenotyping of mice that display traits of bone disease. Two novel mutants, Slc20a2 and $\operatorname{Tram} 2$, have phenotypes including brittle bones and abnormal skull and craniofacial morphologies. The session concluded with discussion on CRISPR-Cas9 technology and its application by Lauryl Nutter (Hospital for Sick Children). Her work has generated numerous mouse lines carrying human disease-associated point mutations and gene knockouts that allow for study on a spectrum of diseases.

\section{Cancer and Immunology}

The Cancer and Immunology session focused on initiation of tumorigenesis and infection as well as novel means to treat disease once it has developed. The session included Tyler Jacks (Massachusetts Institute of Technology), who combined various technologies to study the specific genes and pathways involved in tumorigenesis, specifically in lung cancer. His work includes using CRISPR/Cas9 to mutate genes in developing tumors and studying the effects on tumor growth, and engineering cells to express antigens to study the immune response of early tumors. Beverly Mock (Center for Cancer Research) discussed cancer treatment by presenting work that suggests combined inhibition of MTOR and CDK4/6 pathways may be effective in treating T-cell acute lymphoblastic leukemia and lymphoma.

The topic of disease susceptibility was touched upon by Kendra Williams (NHGRI/NIH), whose work focused on cancer susceptibility and identifying germline variations linked to neuroendocrine differentiation, a marker for developing aggressive prostate cancer. Furthermore, Susheel Bhanu Busi (University of Missouri-Columbia) is investigating the role of gut microbiota in cancer susceptibility. He has found that some families of bacteria, such as Peptococcaceae and Akkermansia muciniphila, positively correlate with tumor multiplicity. Kent Hunter (National Cancer Institute) addressed the topic of phenotypic variation by sharing work on why some women develop highly metastatic breast cancer while others do not. His group found that the gene, Arntl2, contributes to metastasis in estrogenreceptor-negative breast cancer. Although research on treatment of disease is important, much of the recent progress in cancer survival has been due to early treatment and improved diagnostic screening. Such diagnostic screening was discussed by Hui Zong (University of Massachusetts), who uses Mosaic Analysis of Double Markers (MADM) to distinguish between pre-malignant mutant cells versus normal cells. This novel approach could someday be applied to quickly diagnose early forms of malignant cancers like glioma.

Clare Smith (University of Massachusetts Medical School) shared her work using a "dual genome" approach to study interactions between Mycobacterium tuberculosis and its mammalian host. She modeled human genetic variation using 100 recombinant inbred mouse strains with targeted single-gene knockouts obtained from the Collaborative Cross. In parallel, she modeled bacterial genetic variation using $M$. tuberculosis whole genome knockout libraries and clinical isolates. Her results identified QTLs associated with bacterial genes for virulence factors, nutrient acquisition, and oxidative radical generation.

\section{Darla Miller Distinguished Service Lecture}

The Darla Miller Distinguished Service Lectureship recognizes individuals whose work benefits the mouse community as a whole (Barrington et al. 2015). Darla Miller exemplified this kind of service to the community with her involvement in the IMGS for 26 years. This year's award recipient, Kenneth Manly from the University of North Carolina at Chapel Hill, possesses a similar selflessness and community involvement. Since 1988, he has built numerous software programs that many people use in their research. He created Map Manager which is capable of mapping Mendelian and quantitative trait loci with mouse crosses and recombinant inbred lines. He also helped develop WebQTL, which is a platform that makes programs like Map Manager accessible to researchers worldwide. Currently, Manly is writing software for the Collaborative Cross, a project striving to produce recombinant inbred mouse lines with increased genetic diversity. His software for the Collaborative Cross not only manages 
the vast amount of records generated during the production of a recombinant mouse, but also displays the data in such a way that let other researchers access and order their strain of interest with ease. Over the last several decades, Manly has displayed unparalleled innovation in software development and has made his programs accessible to the community as a whole, thereby enabling researchers worldwide to pursue answers to their own set of questions. Manly's contributions truly embody Miller's commitment to bridging service with science, thereby allowing the field of mouse genetics to propel forward.

\section{Verne Chapman Memorial Lecture}

Since 1997, the Verne Chapman Memorial Lecture has been held in honor of its namesake, who was a founding member and Director of Scientific Affairs at the Roswell Park Cancer Institute. His research focused on developing chromosome maps of laboratory mice which were crucial in identifying and studying molecular defects associated with cancer. This year's lecture was given by Joseph Nadeau (Pacific Northwest Research Institute) at the Translational and System Genetics Session. This session covered aspects of inheritance ranging from Mendelian inheritance to epigenetics. Recent advances in the area of epigenetics have dispelled the notion that generations are separate entities. Rather, generations are more continuous than initially thought, as offspring "inherit memories of ancestral genetics and environments." Even the fundamental Mendelian concept of random segregation has been challenged, since factors such as diet and genetic variation can lead to gamete preference at fertilization. Nadeau's lecture highlighted the fact that the foundational concepts of modern scientific dogma can be constantly changed or appended by paradigm-shifting discoveries.

\section{Looking forward}

A major focus of the IMGS is trainee mentorship. However, as in many other scientific fields, the proportion of women mouse geneticists moving into senior-level faculty positions remains much lower than the proportion of women trainees. The IMGS announced two initiatives at this year's business meeting that focus on career development for women. First, the IMGS is establishing an award in honor of Dr. Mary Lyon, a founding member of the society and regular IMGC attendee. This award will honor outstanding junior-level women scientists (pre-tenure assistant professor or equivalent) in the mouse genetics community. The first Mary Lyon Award will be presented in 2018. Second, the IMGS is planning to follow the footsteps of other scientific organizations and establish a public resource with a list of women mouse geneticists. This initiative will encourage the inclusion of more women speakers at future conferences and symposia. More information on the Mary Lyon Award and the public resource can be found on the IMGS website. Individuals who wish to be included on the list should send their name, title, institution, email, website link, and up to ten keywords that describe their research area toimgsgeneral@gmail.com.

Throughout the meeting, IMGS members participated in a cross-disciplinary discussion of large data management and accessibility. Much of the conversation centered upon whether the current system of separate model organismspecific databases best serves an increasingly diverse and collaborative community of researchers. The NHGRI/NIH has recently proposed funding reductions for the Model Organism Databases (MODs) and to merge the individual yeast, worm, fly, fish, and mouse databases into a single platform. While most acknowledge that the databases can be improved, there is concern that the proposed plan may result in the loss of organism-specific datasets. Researchers are also concerned that a reduction in funding may detrimentally affect literature curation and genome annotation within MODs. A letter emphasizing support for MODs received over 10,000 signatures and was presented to the NIH Director, Francis Collins, at the meeting.

Looking ahead, the 31st IMGC will be a joint event with the MMG and the European Molecular Biology Laboratory (EMBL). The conference is titled "Mammalian Genetics and Genomics: From Molecular Mechanisms to Translational Applications" and will be held in Heidelberg, Germany on October 24-27, 2017. More details can be found at http://www.embl.de/training/events/2017/MMM17-01/. This conference will be the only annual meeting for the IMGS and MMG in 2017 and promises to continue the tradition of bringing together scientists that conduct cuttingedge research in mammalian systems through use of molecular, genetic, and genomic approaches. Please consider joining the IMGS at this exciting event and keep an eye on the IMGS website for trainee scholarship information.

Acknowledgements The IMGS would like to thank all members of the Mouse Genetics 2016 organizing committee, which consisted of IMGS Sceretariat members President Teresa Gunn (co-chair), Past President David Beier, Vice President Martin Hrabé de Angelis; MMG organizers Monica Justice (co-chair), Yumiko Saga, Philippe Soriano, and Francois Spitz; and Darla Miller and GSA staff. We extend our gratitude to all IMGS Secretariat members (Viive Howell, Steve Munger, Fernando Pardo-Manuel de Villena, Thomas Keane, Lluis Montoliu, Bill Pavan, Clare Smith, and Gabriela SanchezAndrade) and John Schimenti for reviewing abstracts and providing other invaluable support, and to Monica McAndrews for reviewing the nomenclature of every submitted Mouse Genetics 2016 abstract and for judging nomenclature of trainee posters. The IMGS would also like to acknowledge the sponsors who provided prizes for trainee presentations: the IMGS, Cambridge Journals, AACR, Genomics, 
genesis, Genome Research, and Springer-Verlag. Support for Mouse Genetics 2016 trainee scholarships was provided by NIH grant 2R13HG0002394 to the IMGS from NHGRI and NICHD as well as funding from Mouse News Letter Ltd.

\section{References}

Barrington WT, Morgan AP, Siracusa LD (2015) The 28th International Mammalian Genome Conference-meeting report. Mamm Genome 26:201-209
Gonzales NM, Howell VM, Smith CM (2016) 29th International Mammalian Genome Conference meeting report. Mamm Genome 27:169-178 